\title{
Comparison of the Effectiveness of Cognitive Behavioral Therapy and Neurofeedback: Reducing Insomnia Symptoms
}

\author{
Nooshin Basiri ${ }^{1}$, Zahra Khayyer ${ }^{2}$, Habib Hadianfard ${ }^{3} \&$ Amirhossein Ghaderi ${ }^{4}$ \\ ${ }^{1} \mathrm{PhD}$ candidate of clinical psychology. University of Shiraz, Iran. \\ ${ }^{2} \mathrm{PhD}$ candidate of Psychology, University Of Isfahan, Isfahan, Iran. \\ ${ }^{3}$ Professor of Clinical Psychology, Shiraz University, Shiraz, Iran. \\ ${ }^{4} \mathrm{PhD}$ Candidate of Cognitive Neuroscience, Cognitive Neuroscience Lab. Department of Psychology. University \\ of Tabriz, Tabriz, Iran. \\ Correspondence: Noushin Basiri, PhD candidate of clinical psychology. University of Shiraz. Iran. E-mail: \\ noushin.basiri@gmail.com; And Zahra Khayyer, PhD candidate of Psychology, University Of Isfahan, Isfahan, \\ Iran. E-mail: z.khayer62@yahoo.com
}

Received: January 16, 2017 Accepted: March 20, 2017 Online Published: March 27, 2017

doi:10.5539/gjhs.v9n7p35 URL: https://doi.org/10.5539/gjhs.v9n7p35

\begin{abstract}
Introduction: The term sleep disorder refers to difficulty in initiating sleep, maintaining it or a relaxing sleep despite having enough time to sleep. Cognitive behavioral therapy is a non-drug multi-dimensional treatment that targets behavioral and cognitive factors of this disorder. Some studies have shown that psychiatric and neurological disorders can be distinguished from distinct EEG patterns and neurofeedback can be used to make a change in these patterns. This study aimed to compare the cognitive behavioral therapy and neurofeedback in the treatment of insomnia.
\end{abstract}

Methods: The sample included patients, who had already been diagnosed insomnia by a psychiatrist in Isfahan, Iran. Random sampling was employed to choose the participants. Pittsburg sleep quality index (PSQI) was used for the selection of the participants, too. The sample was included 40 patients who were randomly selected and interviewed. Finally they were divided into 3 groups. Data were analyzed using SPSS. Following the analysis the independent effect of the treatment was significant and one-way ANOVA with post hoc test L.S.D were carried out on CBT and control ( $p=0.001), \mathrm{CBT}$ and neurofeedback therapy $(p=0.003)$, neurofeedback treatment and control $(p=0.001)$.

Results: Results showed a significant difference between the groups. Based on the analysis the two abovementioned treatments, neurofeedback therapy in the first position and cognitive-behavioral therapy in the second position were most effective, and the control group showed the lowest efficiency.

Conclusions: Both treatments were significantly effective, and so we can use both NF and CBT for the treatment of insomnia.

Keywords: neurofeedback, cognitive behavioral therapy, insomnia

\section{Introduction}

The term sleep disorder refers to difficulty in initiating sleep, maintaining it or a relaxing sleep despite having enough time to sleep (Renom-Guiteras et al., 2014). The patients with insomnia have symptoms such as face daily dysfunction problems, fatigue, loss of focus, attention and memory, lower academic achievement, impaired job performance, mood disorders, irritability, leave of absence, loss of motivation and energy, more driving mistakes, stress and worry about the quality of sleep (Ogunbode, Adebusoye, Olowookere, Owolab, \& Ogunniyi, 2014). In addition to the negative effects of insomnia on mental health, recently there are many evidences showing the effect of insomnia on physical health, though. There is no doubt that insomnia can disrupt the internal balance of the body (homoeostasis) which in turn can lead to increase mortality rate among these patients (Molen, Carvalho, Prado, \& Prado, 2014). Insomnia is one of the most common sleep disorders and almost $10 \%$ of the world population reported the experience of chronic or recurrent insomnia disorder. In contrast to the DSM-IV, the new statistic diagnostic manual of mental disorders (DSM-5) does not make a distinction between primary and secondary 
insomnia, therefore diagnosis of primary insomnia has been substituted with insomnia disorder as general (Álamo, Lopez-Muñoz, \& García-García, 2011).

\subsection{Treatments}

Benzodiazepines, non-benzodiazepines drugs and melatonin agonists are the first line drug for insomnia. Secondary pharmacological treatments are included antidepressant, diphenhydramine, anti-psychosis, and barbiturates (Han, Kim \& Chung, 2014). Although these drugs are effective, but they often create a lot of side effects, such as being sleepy during the day, dizziness, mild headache, cognitive impairment, ataxia and dependence (Feyzabadi, Jafari, Feizabadi, Ashayeri, Esfahani, \& Aval, 2014). Pharmacotherapy only improves insomnia for a short period of time (Ramakrishnan, 2007).

According to some studies, another factor may cause insomnia is related to psychological characteristics and personality traits. Considering the research of Regan et al. (2014), insomnia is experienced more by perfectionist personality type. In chronic insomnia, sleeping difficulty is associated with subjective interpretations, beliefs, attitudes and behaviors that perpetuate and worsen sleep disorder (Molen, Carvalho, Prado, \& Prado, 2014). Among non-pharmacological treatments, Cognitive Behavioral Therapy (CBT) is a multi-dimensional treatment that targets behavioral and cognitive factors underling insomnia. Spellman's $3 P$ (Note 1) model of insomnia helps with recognizing the role of negative core beliefs and usual cognitive errors in continuing insomnia symptoms and teaching strategies to fix them (Gosling et al., 2014). On the other hand, in spite of understanding the neurobiological basis of insomnia, the pathophysiology of insomnia is remained unclear. Hyper arousal theory is the most dominant theory explaining insomnia which causes increasing of sensory and affect processing leading to the onset and maintenance of sleeplessness. An ERP study showed that the amplitude of P2 and N1 is greater in people with insomnia than control group which related to greater cortical arousal (Kilgore, Schwab, Kimpman, Deldonno, \& Weber, 2013).

\subsection{Mechanisms}

In addition, fMRI studies demonstrated increased functional connectivity in premotor area, sensory-motor regions, and emotional circuits. Recently, increased correlations between activity of sensory regions and two dominant brain networks; default-mode network (passive or free brain) and the salience network (different cognitive and affective processing) regions, is observed (Zhao, Wang, Zhang, Karama, Khundrakpam, Zhang, \& Evans, 2015). New studies have shown that patients with insomnia have an unusual high activity of beta band power $(32-14 \mathrm{~Hz})$ in their electroencephalography (EEG) (Hammer, Barbara, \& Colbert, 2011) as high frequency waves which related to activity of arousal system. It is in consistent with hyper-arousal theory. Most studies have found that increased beta activity is quite obvious in patients with insomnia compared to people with normal sleep in the initiating moment or during NREM (Note 2). These findings are based on EEG measurements of the central points using 1-channel and 2-channel measurements including the $\mathrm{C} 3, \mathrm{CZ}$ or $\mathrm{C} 4$ and $\mathrm{O} 2$ points (Perlis, Gehrman, Terzano, Cote, \& Riemann, 2010). It was documented in limited number of studies that increased beta waves especially occur in people with insomnia disorder (Figure 1; Perlis, Gehrman, Terzano, Cote, \& Riemann, 2010).

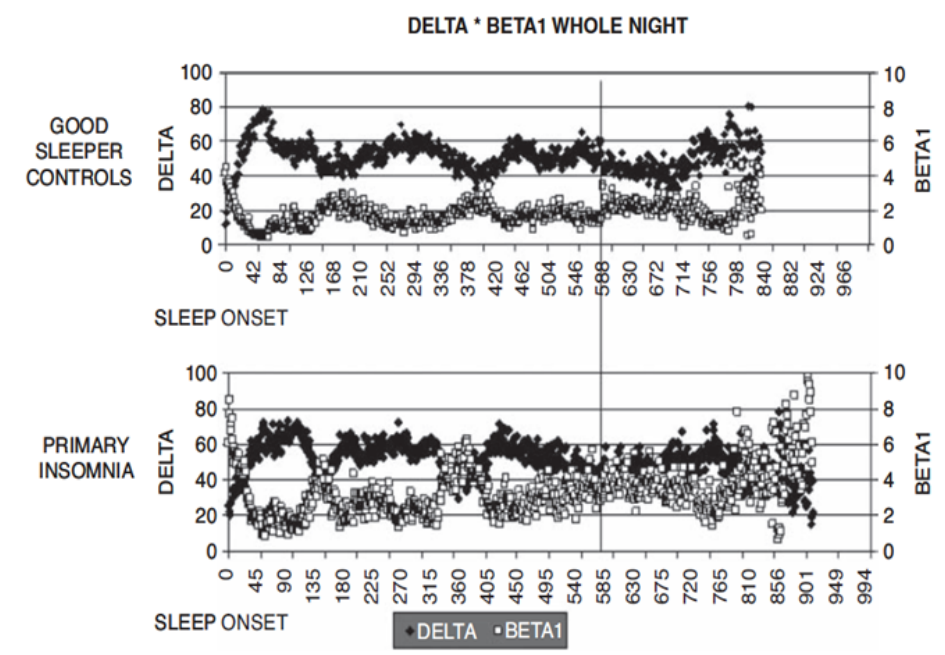

Figure 1. Increased beta waves in individuals with sleeping Disorders 
At the present time, neurotherapists consider neurofeedback as a new avenue for evaluating and changing brain status. Neurofeedback is a kind of biofeedback, which suggests the mutual association of mind and body. Some pieces of research have shown that psychiatric and neurological disorders can be distinguished from distinct EEG patterns (Masterpasqua \& Healey, 2003). In fact, neurofeedback is a method for normalizing the activity of brain using operant conditioning mechanisms (Hammond, 2011). For instance, in Hammer, Colbert, Brown \& Ilioi study (2011), findings showed that neurofeedback had significant effect on improving the quality of sleep and significant decrease were observed in beta waves. The results of the study by Carney \& Edinger (2006) also exhibited that, although neurofeedback training does not affect the subsequent spindle activities, but a growing trend can be seen in the power of Sigma wave width in NREM sleep stage after applying neurofeedback compared with the control group.

\subsection{Aim}

Regarding previous research studies, the current study aimed at comparing the effects of CBT and neuro-feedback in improving sleep quality and reducing insomnia symptoms. No similar research has been performed so far to compare behavioral therapy and neurotherapy in insomnia. As regard to hyper-arousal hypothesis, we use two structurally different treatments to deal with insomnia. CBT, as a behavioral therapy, may be useful to control of arousal system and neurofeedback, as a neural based therapy, can be modulate the neural activity of associated brain regains involved in arousal. So, this hypothesis has been made to address the following issue: Which treatment has better and more significant effects on sleep induction and maintenance.

\section{Materials}

\subsection{Participants}

With regard to the overall goal of this study to compare the effectiveness of cognitive behavioral therapy and neurofeedback on insomnia treatment, this experimental study was performed with a pretest and posttest design with control group. 40 patients who had already been diagnosed insomnia by a psychiatrist (based on medical and sleep histories, physical and psychological tests) were randomly assigned in CBT, neurofeedback, and control groups (age: between 20-40, gender: both male and female).

\subsection{Research Design}

All of the participants completed consent forms for participating in the study and publishing the results. After performing the pretests, first they were randomly assigned to two groups; treatment group $(N=20)$ or control group $(N=20)$, then the treatment group was randomly assigned to two conditions; NF $(N=10)$ or CBT $(N=10)$ using GraphPad Software. The experiment groups participated in therapy sessions for three months. The sessions were held 3 days a week and lasted forty-five-minutes for neuro-feedback group (a total of 20 sessions) according to Hammer's protocol (2011) presented in his reported article. Patients attended to the cognitive behavioral therapy group once a week (a total of 9 sessions) based on Savard's protocol introduced in the report of his research (Savard, Simard, Ivers, \& Morin, 2005). Fortunately there was no dropout during the study.

\subsubsection{Inclusion/Exclusion Criteria}

Participant's qualified for the study if they met the diagnostic criteria for insomnia disorder according to the Diagnostic and Statistical Manual of Mental Disorders of the American Psychiatric Association, 5th edition (DSM-5) and also had following criteria;

1- Ages between 20 to 40 years old 2- their Scored on the PSQI be more than 5. 3- Were free of other mental disorders as determined in-depth psychological screening interview 4- Were not taking prescription medication for insomnia disorder 5- confirm to be available for the 3 months the study might take.

\subsection{Interventions}

The procedure was in a way that every session, SMR wave $(12-15 \mathrm{~Hz})$-as increases in insomnia-was reinforced and waves of 2-7 Hz and also $20-30 \mathrm{~Hz}$ were suppressed. This procedure was carried out in bipolar way on $\mathrm{C} 3-\mathrm{C} 4$ (according to 10-20 electrode arrangement). After 20 sessions, posttests were applied in three groups with an interval of one week. CBT was provided by a therapist who was staff psychologists of the Department of Clinical Psychology (University of Isfahan) and had completed her 1-year full-time CBT training. CBT sessions are presented in Table 1. 
Table 1. Description of Cognitive Behavioral Therapy Sessions

\begin{tabular}{ll}
\hline Session & Goals \\
\hline $\mathbf{1}$ & $\begin{array}{l}\text { Introduction, statement of the objectives, process of sessions, pretest implementation, discussion on proper sleep } \\
\text { and its features, providing information about the effects of sleep on mental and physical health }\end{array}$ \\
$\mathbf{2}$ & $\begin{array}{l}\text { Defining Insomnia, providing statistics and information about proper sleep and insomnia, reviewing concern as } \\
\text { the main component of insomnia }\end{array}$ \\
$\mathbf{3}$ & Evaluation of various concerns, and incorrect insights pertaining to sleep and insomnia \\
$\mathbf{4}$ & Training sleep hygiene and requirements for good sleep \\
$\mathbf{5}$ & training sleep restriction and meditation techniques \\
$\mathbf{6}$ & Describing concept of conditioning and its role in insomnia, stimulus control training \\
$\mathbf{7}$ & Providing guidelines to deal with insomnia in sleep place and relaxation training \\
$\mathbf{8}$ & Continuing relaxation training and troubleshooting \\
$\mathbf{9}$ & Investigation of problems and implementing posttest \\
\hline
\end{tabular}

\subsection{Measures}

Pittsburgh Sleep Quality Index (PSQI) Questionnaire: The PSQI is one of the most common and important questionnaires all over the world. This questionnaire examines the quality of sleep. PSQI measures seven domains: subjective sleep quality, sleep latency, sleep duration, sleep efficiency, sleep disorders, use of sleep medication, and daytime dysfunction over the last month. Scoring is based on a three point likert scale. The score of 0 represents normal state, 1 represents a mild problem, 2 illustrates a moderate problem, and 3 is demonstrative of a severe problem in sleeping (Morin, Bootzin, Buysse, Edinger, Espie, \& Lichstein, 2006). It takes 5-10 minutes to fill out this questionnaire .The reliability of this questionnaire is 89.5 , respectively (Molen, Carvalho, Fernandes, $\&$ Fernandes Prado, 2014). Haidari's study, indicated a high degree of internal consistency (Cronbach's a of 0.83), with the strongest correlations in Sleep Efficiency and Subjective Sleep Quality (Heydari, Ehtesham Zade, \& Marashi, 2011).The test-retest reliability of global scores is 0.85 . Validity is supported by its ability to distinguish insomnia patients from controls, and to a lesser extent, concurrent validity with polysomnography (Buysse, Reynolds, Monk, Berman, \& Kupfer, 1989).

Insomnia Severity Index: The ISI is a 7-item questionnaire designed to evaluate insomnia severity on the basis of (1) difficulties falling asleep, (2) nighttime awakenings, (3) early morning awakenings, (4) impairment of daytime functioning due to sleep problems, (5) noticeability of impairments, (6) distress or worry caused by sleep difficulties, and (7) current dissatisfaction with sleep. Each item is rated using a 5-point Likert scale ranging from 0 (not at all) to 4 (extremely). The total score is obtained by adding ratings of the seven items (possible range: 0 28). (Blais, Gendron, Mimeault, \& Morin, 1997). Cronbach's alpha of 0.74 to 0.78 is reported in studies. The ISI has good face validity with the concept of insomnia as defined by DSM-5. Formal evaluation of content validity was demonstrated via principal component analysis, which yielded 3 components (impact, severity, and satisfaction). These components are consistent with the diagnostic criteria of insomnia and captured $72 \%$ of the variance (Wegener \& Smith, 2003).

Neurofeedback: In neurofeedback training, the PROCAMP-2 system which is made in Canada and Biography Infinity software were used.

\subsection{Statistical Analysis}

Assessing the effects of neurofeedback and CBT, analyses were performed and research hypothesis was examined. All statistical analyses were carried out using the version 20.0 of the Statistical Package for Social Sciences (SPSS) software. To identify differences between groups at pre/post-test, Multivariate Analysis Of Variance (MANOVA) was used. Pretest assumed as covariance in our analysis to control its probable effect. Furthermore, we analyzed the data of a random sample $(\mathrm{N}=4)$ using repeated measure ANOVA with one within subject factor just for SMR trends checking.

\subsection{Ethics}

The study was approved by the Ethics Committee of the Faculty of Educational Sciences and Psychology (University of Isfahan) and there was no fund for the study. 


\section{Results}

Demographic characteristics of the participants are presented in Table 2, 25 percent of the participants were female and 75 percent were male. All of them lived in Isfahan city. Descriptive statistics of PSQI \& ISI are presented in Table 3.

Table 2. Statistics of demographic features

\begin{tabular}{llll}
\hline & & N & Percentage \\
\hline \multirow{2}{*}{ gender } & men & 15 & 37.5 \\
& women & 25 & 62.5 \\
\hline \multirow{2}{*}{ age } & $20-30$ & 18 & 45 \\
& $30-40$ & 12 & 55 \\
\hline \multirow{2}{*}{ Educational degree } & Diploma(high school degree) & 8 & 20 \\
& BA/BS & 26 & 65 \\
& MA/MS & 6 & 15 \\
\hline
\end{tabular}

Table 3. Descriptive statistics of PSQI pre-test

\begin{tabular}{llll}
\hline & Group & Mean & Standard deviation \\
\hline \multirow{3}{*}{ PSQI } & Neurofeedback & $15 / 2$ & $2 / 6$ \\
& C.B.T & $14 / 9$ & $2 / 6$ \\
& control & $15 / 05$ & $2 / 5$ \\
\hline \multirow{3}{*}{ ISI } & Neurofeedback & $5 / 4$ & $5 / 08$ \\
& C.B.T & $9 / 9$ & $1 / 9$ \\
& control & $16 / 7$ & $2 / 5$ \\
\hline
\end{tabular}

As demonstrated in Table 3, the mean score of PSQI in post-test was decreased in neurofeedback group ( $M=5.4$, $\mathrm{Sd}=5.08)$ and $\mathrm{CBT}(\mathrm{M}=9.9, \mathrm{Sd}=1.9)$, no changes was seen in control group though $(\mathrm{M}=16.7, \mathrm{Sd}=2.5)$. To test the research hypothesis, independent one-way ANOVA (pretest as covariance variable) was used. The normality of the data and the equality of variances were examined respectively according to QQ Plot chart and Levine's test. to establish the required preconditions for the analysis of variance (Figure 2). The results illustrated normal distribution of the data and the Levine's test $(p<0.4)$ indicated equal variances.

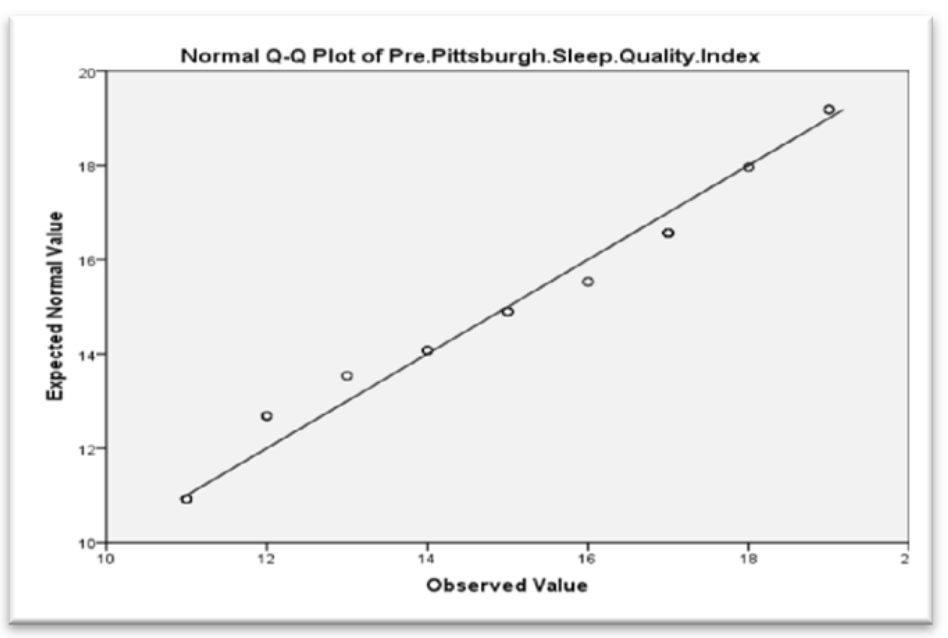

Figure 2. The test of Normality 
Table 4. Results of the one way ANOVA test with pretest factor as covariance

\begin{tabular}{lllllll}
\hline & SS & DF & MS & F & sig & ${\text { Partial } \boldsymbol{\eta}^{2}}^{2}$ \\
\hline treatment & $169 / 23$ & 2 & $84 / 61$ & $9 / 83$ & $0 / 001$ & $0 / 36$ \\
Pre-test & $25 / 35$ & 1 & $25 / 35$ & $2 / 9$ & $0 / 09$ & $0 / 09$ \\
Treatment-pre test & $78 / 57$ & 2 & $39 / 28$ & $4 / 56$ & $0 / 01$ & $0 / 2$ \\
Error & $292 / 53$ & 34 & $8 / 6$ & & & \\
total & 7235 & 40 & & & & \\
\hline
\end{tabular}

In Table 4, considering the significance of the interaction between the dependent variable and pretest (variable covariance) $(p<018)$, the data does not support the assumption of homogeneity of slopes of regression and the pretest factor cannot be considered as covariance. On the other hand, the relationship between pretest and the dependent variable based on Figure 3 for dispersion is not linear. As a result, it cannot be considered as a covariate.

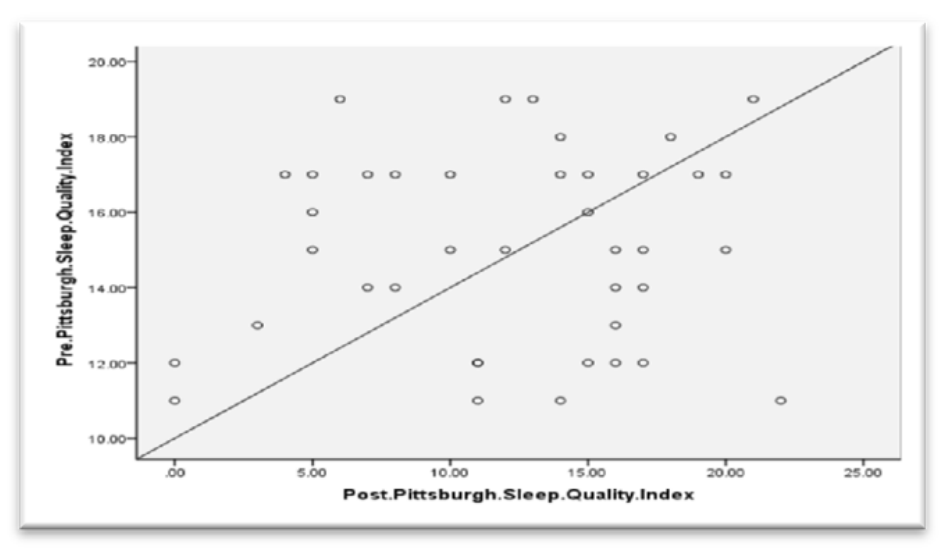

Figure 3. Relationship between pretest and the dependent variable

Table 5. Results of one way ANOVA

\begin{tabular}{lllllll}
\hline & SS & DF & MS & F & sig & Partial $\mathbf{~}^{2}$ \\
\hline Between subject & $923 / 33$ & 2 & $461 / 66$ & $44 / 78$ & $0 / 001$ & $0 / 71$ \\
Error & $371 / 10$ & 36 & $10 / 3$ & & & \\
total & 7235 & 40 & & & & \\
\hline
\end{tabular}

According to one-way ANOVA, the mean value of PSQI has been statistically significant between three groups ( $F^{2}$, $36=44.78$; Partial $\eta^{2}=0.36 ; p=0.001$ ) (Table 5). Post hoc L.S.D, between-group comparisons (see Table 6) revealed significant differences between cognitive-behavioral therapy and control $(p=0.001)$, between cognitive-behavioral therapy and neurofeedback treatment $(p=0.003)$, and between neurofeedback treatment and control $(p=0.001)$ groups. Considering aforementioned descriptive statistics, neurofeedback treatment $(M: 5.4$, $S D$ : 5.08) had the most effectiveness; cognitive-behavioral therapy (M: 9.9, $S D: 1.9)$ was in the second place; whereas the control group ( $M: 12.17, S D: 5.7)$ showed the least effectiveness (Figure 4).

Table 6. Two by two comparison of the means based on LSD statistic

\begin{tabular}{llll}
\hline Groups & Means differences & Standard deviation & Sig \\
\hline CBT-neurofeedback & $-4 / 5$ & $1 / 4$ & $0 / 003$ \\
CBT-control & $-6 / 7$ & $1 / 2$ & $0 / 001$ \\
Neurofeedback-control & $-11 / 3$ & $1 / 2$ & $0 / 001$ \\
\hline
\end{tabular}




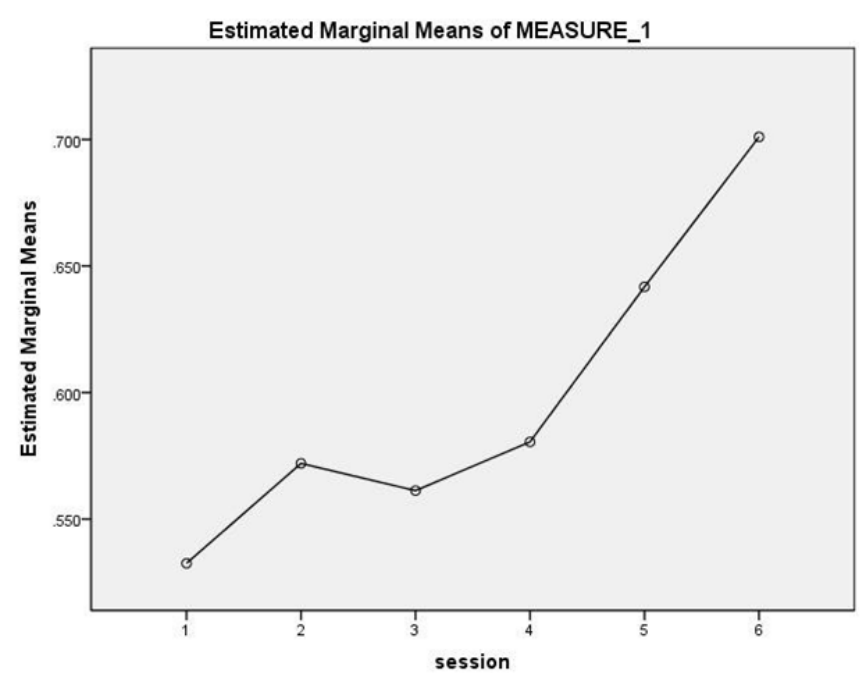

Figure 4. Comparison of the mean scores of sleep quality for the three treatment groups (with the highest average belonging to neurofeedback group)

According to one-way ANOVA result, insomnia severity decreased. Neurofeedback $(15.5 \pm 2.8)$ showed more decline in severity than cognitive-behavioral therapy $(16.5 \pm 3.2)$ and control group (19.2 \pm 4.2$)$. Table 7 showed significant differences between three conditions $(\mathrm{p}<.001)$. Pairwise comparisons were statistically significant for all conditions.

Table 7. Results of the one way ANOVA test for ISI

\begin{tabular}{lllllll}
\hline & SS & DF & MS & F & sig & Partial $\eta^{2}$ \\
\hline Treatment & 102.2 & 2 & 51.1 & 70.7 & .001 & .8 \\
Pre-test & 472.2 & 1 & 472.2 & 653.5 & .001 & .9 \\
Error & 26.01 & 36 & $8 / 6$ & & & \\
total & 12996 & 40 & & & & \\
\hline
\end{tabular}

SMR changes were checked for 4 patients (as a random sample) after each 2 sessions for total 12 sessions in order to make sure of accurate applying of NF. According to table 8 a significant accelerating trend of beta-SMR was observed during neurofeedback training in order to decreasing motor cortex hyper-arousal. Repeated measure ANOVA with one within subjects factor revealed that there were significant differences among the mean values of the six sessions $\left(\mathrm{F}(5,15)=5.368 . \mathrm{P}<0.005, \eta^{2}=0.65\right)$.

Table 8. Test of within subject effects of repeated measure analysis of variance of SMR trends during 12 sessions of neurofeedback training

\begin{tabular}{lllllll}
\hline Source & Sum of squares & df & Mean square & F & sig & Partial Eta square \\
\hline (within subjects) sessions & 0.007 & 5 & 0.015 & 5.368 & 0.005 & 0.642 \\
\hline Error(sessions) & 0.043 & 15 & 0.003 & & & \\
\hline
\end{tabular}

As Figure 5 shows, the level of SMR generally increased with the numbers of neurofeedback training sessions as predicted by our hypotheses (Figure 5). 


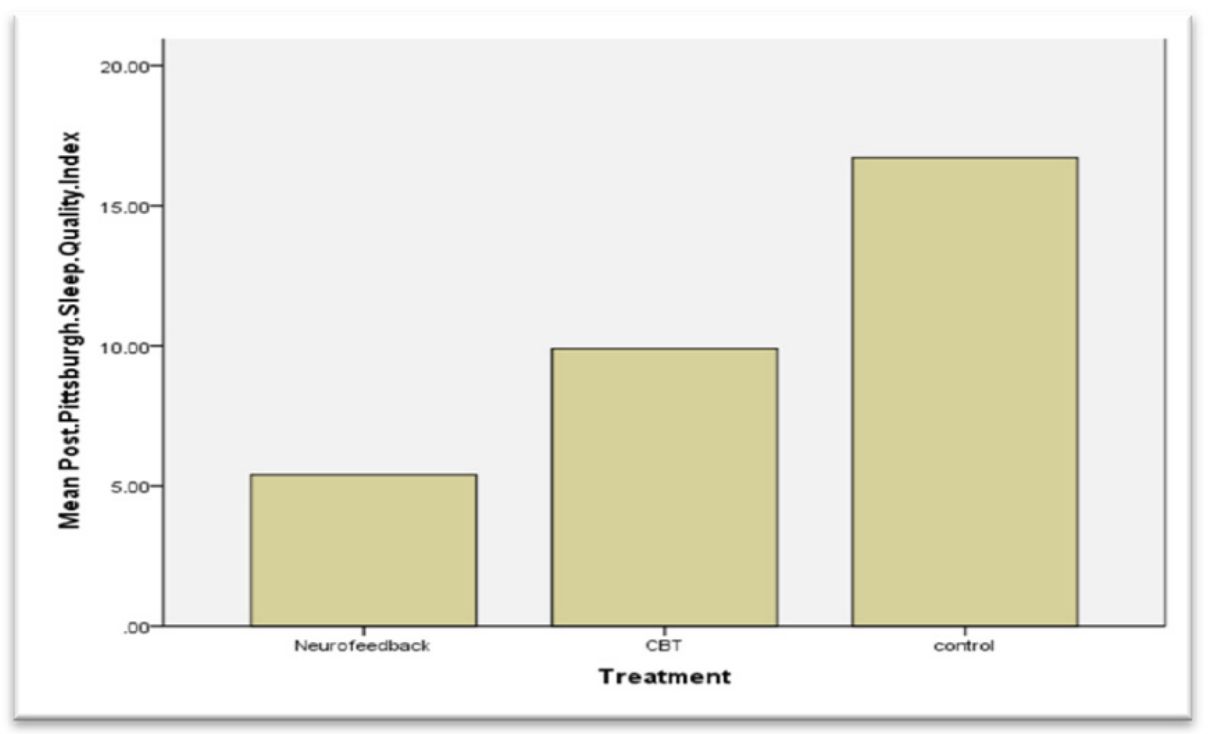

Figure 5. Visual inspection of increasing the SMR trends

\section{Discussion}

This study aimed to evaluate and compare the neurofeedback therapy and cognitive behavioral therapy improving the symptoms of insomnia. The results showed that, of the selected treatments, neurofeedback was more effective in decreased symptoms of insomnia and increased the quality of sleep. As far as we know, no study has compared $\mathrm{NF}$ and CBT. However, the present study showed the same results concerning CBT on ameliorating the symptoms of insomnia as previous studies such as; Edinger and Sampson (2003); Morin, Bootzin, Buysse, Edinger, Espie, and Lichstein (2006); Smith, Huang and Manber (2005); Morin, Jarvis and Lynch (2007). Furthermore, our findings are consistent with some studies which applied NF as a treatment for insomnia like Berner, Schabus, Wienerroither and Klimesch (2006); Cortoos, Verstraeten and Cluydts (2006); Cortoos, Valck, Arns, Breteler and Cluydts (2010) and Hammer, Colbert, Brown and Ilioi (2011).

We targeted $\mathrm{C} 3$ and $\mathrm{C} 4$, as the two critical points on the sensory motor area, for neurofeedback training. It has frequently shown that increase of Sensory Motor Rhythms in central regions of the skull was associated with decreased activity of these areas (Arns \& Kenemans, 2014; Arns, Ridder, Strehl, Bretler, \& Coenen, 2009). Inversely, hypo-activity of SMR is related to hyper activation of associated sensory motor regions in people who suffering from ADHD (Arns, Heinrich, \& Strehl, 2013; Moriyama, Polanczy, Caye, Banaschewski, Bbrandeis, \& Rohde, 2012), anxiety (Hammond, 2005, 2011; Gruzelier, Tompson, redding, Brandt, \& Steffert, 2013) and some other kind of disorders which related to arousal system hyper-activity, such as insomnia (Hammer, Colbert, Kimberly, \& Ilioi, 2011; Arns \& Kenemans, 2012; Cortoos, Valck, Arns, Breteler, \& Cludyts, 2009). Activity of high frequencies band power (including $20-30 \mathrm{~Hz}$ ), also, represents hyper-activity of associated sensory motor areas (Marzbani, Marateb, \& Mansourian, 2016).

According to literature review, the activity of $2-6 \mathrm{~Hz}$ was directly related to cortical activity and reducing of these frequencies may be associated with hypo-activity of a selected region (Pesaran, Rezaris, Sahani, Mitra, \& Andersen, 2000). With regard to previous findings about hyper activity of sensory motor areas in insomnia (Hammer, Colbert, Kimberly, \& Ilioi, 2011; Arns \& Kenemans, 2012; Cortoos, Valck, Arns, Breteler, \& Cludyts, 2009) which results in modulation of arousal system, we decided to increase the level of SMR and decrease of 2-6 $\mathrm{Hz}$ and $20-30 \mathrm{~Hz}$ in $\mathrm{C} 3$ and $\mathrm{C} 4$, simultaneously. This protocol can decrease the activity of sensory motor areas as it is mentioned above In general, it can be concluded that using neurofeedback can be considered as a therapeutic alternatives for patients with sleeping disorders.

Neurofeedback treatments lead to significant SMR increase in C3 and C4. In 7 of 10 patients increased beta trend was observed. Beta enhancement level was positively correlated with an improvement in sleep initiation and maintenance. Thus, according to hyper arousal theory and hyper metabolism of the motor cortex (Nofzinger, Buysse, Germain, Price, Miewald, \& Kupfer, 2015), we may conclude that increasing SMR at C3 and C4 is an efficacious NF protocol in insomnia. This reduction is specifically occurred in the Rolandic fissure (central sulcus) and sensory motor cortex. Our results showed a significant decreasing trend in high frequencies $(20-30 \mathrm{~Hz}) \mathrm{which}$ 
supports the study hypothesis. However, there was no significant decrease in amplitude of 2-6 Hz.

In addition, CBT has shown to be efficient as treatment of insomnia (references) by correcting patients' unrealistic expectations, impaired perception and concerns. Inaccurate or biased expectations may, in turn, cause stress and maladaptive behaviors in contrast to encourage calm and peaceful thoughts before going to sleep. Research demonstrated that people with sleep disorders often have automatic negative thoughts triggered by unpleasant emotions at night that result in dysfunctional beliefs about sleep. Thus, in cognitive behavioral therapy, patients learn how to take control of their interpretations of thoughts and feelings using different techniques such as behavioral experiments, thoughts records, and imagery-based exposures and so on in order to decrease insomnia symptoms.

\section{Conclusions}

Finally, changes in consciousness are actually representative of changes in brain and neurofeedback as a therapeutic approach is based on targeting brain waves. Attempt to change the brain waves through methods including neurofeedback treatment leads to changes in the brain cortex. Also, any change in the electrical activity of the brain following NF, results in the reorganization of the entire electrical environment system which, in turn, creates a comprehensive, natural, and reflexive normalization reaction in the brain that leads to its recovery. Therefore, association between changes in brain waves and conciseness changes is not a mutual linear correlation. In other words, alternation in one of these components does not lead to alternations in the other one. Although, mechanism of plasticity in brain is not clear, the improvement can be observed in behavior which is understandable and measurable to us.

Consequently, there is an association between EEG and subthalamocortical pathways which are responsible for brain rhythms and EEG frequencies. Sleep disorders change these rhythms and neurofeedback procedure makes clinical after effects by readjusting and correcting these rhythms. However, most clinical guidelines for the treatment of insomnia disorder introduced cognitive behavioral therapy as the first line treatment in insomnia with long-term benefits and outcomes.

\section{Acknowledgements}

The authors are thankful to all participants for their patience and cooperation.

\section{Competing Interests Statement}

The authors declare that there is no conflict of interests regarding the publication of this paper.

\section{References}

Álamo, C., López-Mu-oz, F., \& García-García, P. (2011). Exploring New Frontiers for the Pharmacological Treatment of Insomnia. Clin Exp Pharmacol 4: e133. Doi: 10.4172/2161-1459.1000 e133 Page 2 of 4 Volume 4 Issue 5 1000e133 Clin Exp Pharmacol ISSN: 2161-1459 CPECR, an open access journal aspect of insomnia, the time to fall asleep. In addition, in only one of the three studies carried out in the natural setting was there a difference in the time taken to fall asleep between patients taking ramelteon and those taking placebo. When other aspects of sleep... October of. Retrieved from https://pdfs.semanticscholar.org/3708/52644a7b2cfe57f598267fc67b37b3fc8886.pdf.

Arns, M., \& Kenemans, J. L. (2014). Neurofeedback in ADHD and insomnia: vigilance stabilization through sleep spindles and circadian networks. Neuroscience \& Biobehavioral Reviews, 44, 183-194. https://doi.org/10.1016/j.neubiorev.2012.10.006

Arns, M., de Ridder, S., Strehl, U., Breteler, M., \& Coenen, A. (2009). Efficacy of neurofeedback treatment in ADHD: the effects on inattention, impulsivity and hyperactivity: a meta-analysis. Clinical EEG and neuroscience, 40(3), 180-189. https://doi.org/10.1177/155005940904000311

Arns, M., Heinrich, H., \& Strehl, U. (2014). Evaluation of neurofeedback in ADHD: the long and winding road. Biological psychology, 95, 108-115. https://doi.org/10.1016/j.biopsycho.2013.11.013

Berner, I., Schabus, M., Wienerroither, T., \& Klimesch, W. (2006). The significance of sigma neurofeedback training on sleep spindles and aspects of declarative memory. Applied psychophysiology and biofeedback, 31(2), 97-114. https://doi.org/10.1007/s10484-006-9013-7

Blais, F. C., Gendron, L., Mimeault, V., \& Morin, C. M. (1997). Evaluation de l'insomnie: Validation de trois questionnaires. L'Encéphale: Revue de psychiatrie clinique biologique et thérapeutique. Retrieved from http://psycnet.apa.org/psycinfo/1997-39030-007.

Buysse, D. J., Reynolds, C. F., Monk, T. H., Berman, S. R., \& Kupfer, D. J. (1989). The Pittsburgh Sleep Quality 
Index: A new instrument for psychiatric practice and research. Psychiatry research, 28(2), $193-213$. https://doi.org/10.1016/0165-1781(89)90047-4

Carney, C. E., \& Edinger, J. D. (2006). Identifying critical beliefs about sleep in primary insomnia. Sleep, 29(4), 444-453. Retrieved from https://doi.org/10.1093/sleep/29.3.342

Cortoos, A., De Valck, E., Arns, M., Breteler, M. H., \& Cluydts, R. (2010). An exploratory study on the effects of tele-neurofeedback and tele-biofeedback on objective and subjective sleep in patients with primary insomnia. Applied psychophysiology and biofeedback, 35(2), 125-134. https://doi.org/10.1007/s10484-009-9116-z

Cortoos, A., De Valck, E., Arns, M., Breteler, M. H., \& Cluydts, R. (2010). An exploratory study on the effects of tele-neurofeedback and tele-biofeedback on objective and subjective sleep in patients with primary insomnia. Applied psychophysiology and biofeedback, 35(2), 125-134. https://doi.org/10.1007/s10484-009-9116-Z

Cortoos, A., Verstraeten, E., \& Cluydts, R. (2006). Neurophysiological aspects of primary insomnia: implications for its treatment. Sleep medicine reviews, 10(4), 255-266. https://doi.org/10.1016/j.smrv.2006.01.002

Edinger, J. D., \& Sampson, W. S. (2003). A primary care" friendly" cognitive behavioral insomnia therapy. SLEEP-NEW YORK THEN WESTCHESTER-, 26(2), 177-184. Retrieved from https://www.researchgate.net/profile/William_Sampson2/publication/10816262_A_primary_care_\%27frien dly\%27_cognitive_behaviour_insomnia_therapy/links/0fcfd50ba6bddb2c24000000/A-primary-care-friendl y-cognitive-behaviour-insomnia-therapy.pdf.

Fernández, T., Harmony, T., Fernández-Bouzas, A., Díaz-Comas, L., Prado-Alcalá, R. A., Valdés-Sosa, P., ... \& Aubert, E. (2007). Changes in EEG current sources induced by neurofeedback in learning disabled children. An exploratory study. Applied psychophysiology and biofeedback, 32(3-4), 169-183. https://doi.org/10.1007/s10484-007-9044-8

Feyzabadi, Z., Jafari, F., Feizabadi, P. S., Ashayeri, H., Esfahani, M. M., \& Aval, S. B. (2014). Insomnia in Iranian traditional medicine. Iranian Red Crescent Medical Journal, 16(3). Retrieved from https:/www.ncbi.nlm.nih.gov/pmc/articles/PMC4005448/.

Gosling, J. A., Glozier, N., Griffiths, K., Ritterband, L., Thorndike, F., Mackinnon, A., ... \& Christensen, H. (2014). The GoodNight study - online CBT for insomnia for the indicated prevention of depression: study protocol for a randomised controlled trial. Trials, 15(1), 56. https://doi.org/10.1186/1745-6215-15-56

Gruzelier, J. H., Thompson, T., Redding, E., Brandt, R., \& Steffert, T. (2014). Application of alpha/theta neurofeedback and heart rate variability training to young contemporary dancers: State anxiety and creativity. International Journal of Psychophysiology, 93(1), 105-111. https://doi.org/10.1016/j.ijpsycho.2013.05.004

Hammer, B. U., Colbert, A. P., Brown, K. A., \& Ilioi, E. C. (2011). Neurofeedback for insomnia: A pilot study of Z-score SMR and individualized protocols. Applied psychophysiology and biofeedback, 36(4), 251-264. https://doi.org/10.1007/s10484-011-9165-y

Hammond, D. C. (2005). Neurofeedback with anxiety and affective disorders. Child and adolescent psychiatric clinics of North America, 14(1), 105-123. https://doi.org/10.1016/j.chc.2004.07.008

Hammond, D. C. (2011). What is neurofeedback: An update. Journal of Neurotherapy, 15(4), $305-336$. https://doi.org/10.1080/10874208.2011.623090

Han, K. H., Kim, S. Y., \& Chung, S. Y. (2014). Effect of acupuncture on patients with insomnia: study protocol for a randomized controlled trial. Trials, 15(1), 403. https://doi.org/10.1186/1745-6215-15-403

Heydari, A., Ehtesham Zadeh, P., \& Marashi, M. (2011). The relationship between insomnia severity, sleep quality, drowsiness, psychological disorder with educational function in girls. Faslname Zan va Farhang, 1(4), 65-77. Retreived from http://fa.journals.sid.ir/ViewPaper.aspx?id=160413

Killgore, W. D., Schwab, Z. J., Kipman, M., DelDonno, S. R., \& Weber, M. (2013). Insomnia - related complaints correlate with functional connectivity between sensory - motor regions. Neuroreport, 24(5), 233-240. https://doi.org/10.1097/WNR.0b013e32835edbdd

Marzbani, H., Marateb, H. R., \& Mansourian, M. (2016). Neurofeedback: a comprehensive review on system design, methodology and clinical applications. Basic and clinical neuroscience, 7(2), 143. https://doi.org/10.15412/J.BCN.03070208

Masterpasqua, F., \& Healey, K. N. (2003). Neurofeedback in Psychological Practice. Professional Psychology: Research and Practice, 34(6), 652. https://doi.org/10.1037/0735-7028.34.6.652 
Molen, Y. F., Carvalho, L. B. C., Prado, L. B. F. D., \& Prado, G. F. D. (2014). Insomnia: psychological and neurobiological aspects and non-pharmacological treatments. Arquivos de neuro-psiquiatria, 72(1), 63-71. https://doi.org/10.1590/0004-282X20130184

Morin, A. K., Jarvis, C. I., \& Lynch, A. M. (2007). Therapeutic Options for Sleep - Maintenance and Sleep Onset Insomnia. Pharmacotherapy: The Journal of Human Pharmacology and Drug Therapy, 27(1), 89-110. https://doi.org/10.1592/phco.27.1.89

Morin, C. M., Bootzin, R. R., Buysse, D. J., Edinger, J. D., Espie, C. A., \& Lichstein, K. L. (2006). Psychological and behavioral treatment of insomnia: update of the recent evidence (1998-2004). SLEEP-NEW YORK THEN WESTCHESTER, 29(11), 1398. http://216.21.56.228/Resources/PracticeParameters/Review_Insomnia.pdf.

Moriyama, T., Polanczyk, G., Caye, A., Banaschewski, T., Brandeis, D., \& Rohde, L. (2012). Evidenced-based information on clinical use of neurofeedback for ADHD. Neurotherapeutics (springer). Retrieved from https://www.ncbi.nlm.nih.gov/pubmed/22930416

Nofzinger, E. A., Buysse, D. J., Germain, A., Price, J. C., Miewald, J. M., \& Kupfer, D. J. (2004). Functional neuroimaging evidence for hyperarousal in insomnia. American Journal of Psychiatry, 161(11), $2126-2128$. https://doi.org/10.1176/appi.ajp.161.11.2126

Ogunbode, A. M., Adebusoye, L. A., Olowookere, O. O., Owolabi, M., \& Ogunniyi, A. (2014). Factors associated with insomnia among elderly patients attending a geriatric centre in Nigeria. Current gerontology and geriatrics research, 2014. https://doi.org/10.1155/2014/780535

Perlis, M., Gehrman, P.,Terzano, M., Cote, K., \& Riemann, D. (2010). Sleep EEG in Patients With Primary Insomnia. In M. J. Sateia (Ed.), Insomnia: Diagnosis and Treatment (pp. 50-64). London: Informa healthcare. https://doi.org/10.3109/9781420080803.006

Pesaran, B., Pezaris, J. S., Sahani, M., Mitra, P. P., \& Andersen, R. A. (2002). Temporal structure in neuronal activity during working memory in macaque parietal cortex. Nature neuroscience, 5(8), 805-811. https://doi.org/10.1038/nn890

Ramakrishnan, K. (2007). Treatment options for insomnia. South African Family Practice, 49(8), 34-41. Retrieved from http://www.ajol.info/index.php/safp/article/view/13398

Regen, W., Hertenstein, E., Weil, P., Kyle, S. D., Holz, J., Baglioni, C., ... \& Spiegelhalder, K. (2015). Perfectionistic Tendencies in Insomnia Patients' Behavior During Psychometric Testing. Behavioral sleep medicine, 13(5), 387-394. https://doi.org/10.1080/15402002.2014.919918

Renom-Guiteras, A., Planas, J., Farriols, C., Mojal, S., Miralles, R., Silvent, M. A., \& Ruiz-Ripoll, A. I. (2014). Insomnia among patients with advanced disease during admission in a Palliative Care Unit: a prospective observational study on its frequency and association with psychological, physical and environmental factors. BMC palliative care, 13(1), 40. https://doi.org/10.1186/1472-684X-13-40

Savard, J., Simard, S., Ivers, H., \& Morin, C. M. (2005). Randomized study on the efficacy of cognitive-behavioral therapy for insomnia secondary to breast cancer, part I: Sleep and psychological effects. Journal of Clinical Oncology, 23(25), 6083-6096. https://doi.org/10.1200/JCO.2005.09.548

Shahrifar, M. (2010). The effect of relaxation on sleep quality and drowsiness of nurses. MD theses. Jondi Medical University of Jondi Shapoor (persion).

Smith, M. T., \& Wegener, S. T. (2003). Measures of sleep: the insomnia severity index, medical outcomes study (MOS) sleep scale, Pittsburgh sleep diary (PSD), and Pittsburgh sleep quality index (PSQI). Arthritis Care \& Research, 49(S5). https://doi.org/10.1002/art.11409

Smith, M. T., Huang, M. I., \& Manber, R. (2005). Cognitive behavior therapy for chronic insomnia occurring within the context of medical and psychiatric disorders. Clinical psychology review, 25(5), 559-592. https://doi.org/10.1016/j.cpr.2005.04.004

Trivedi, M. H., Rush, A. J., Wisniewski, S. R., Nierenberg, A. A., Warden, D., Ritz, L., ... \& Shores-Wilson, K. (2006). Evaluation of outcomes with citalopram for depression using measurement-based care in STAR* D: implications for clinical practice. American journal of Psychiatry, 163(1), 28-40. https://doi.org/10.1176/appi.ajp.163.1.28

Zhao, L., Wang, E., Zhang, X., Karama, S., Khundrakpam, B., Zhang, H. \& Evans, A. C. (2015). Cortical structural connectivity alterations in primary insomnia: Insights from MRI-based morphometric correlation analysis. BioMed research international, 2015. https://doi.org/10.1155/2015/817595 


\section{Notes}

Note 1. Spielman Model: The 3P model suggests that insomnia is perpetuated by sleep extension and thus should be managed with treatment protocols that restrict time in bed (i.e., compress the sleep period). One implication for treatment is that sleep compression need not occur in a radical fashion, but could be accomplished over days or weeks.

Note 2. Non-rapid eye movement sleep.

\section{Copyrights}

Copyright for this article is retained by the author(s), with first publication rights granted to the journal.

This is an open-access article distributed under the terms and conditions of the Creative Commons Attribution license (http://creativecommons.org/licenses/by/4.0/). 